\title{
Geoquímica de elementos mayores de los cuerpos intrusivos terciarios de Tierra Caliente, Edo. de México, México
}

\author{
Eduardo González-Partida* y Luis F. Vassallo \\ Unidad de Investigación en Ciencias de la Tierra, Instituto de Geología, \\ Universidad Nacional Autónoma de México, Campus UNAM - Juriquilla, \\ A.P. 1-742, Querétaro, Qro. C.P. 76230 \\ *egp@unicit.unam.mx
}

\section{Resumen}

El magmatismo intrusivo postorogénico localizado en la región de Tierra Caliente, Edo. de México, a $~ 350 \mathrm{~km}$ de la paleotrinchera del Eoceno-Mioceno, se emplazó en una corteza continental de $35 \mathrm{~km}$ de espesor, caracterizada por esquistos negros y rocas volcanosedimentarias cretácicas.

La composición individual de los stocks varía de adamelitas a granitos de biotita. Pertenecen al dominio peraluminoso mesocrático; asociación aluminosa con un índice de aluminosidad alto y, por su rango de alcalinidad, pertenecen al subtipo sódico. Estas características geoquímicas son muy similares a las determinadas en la serie Tizapa (esquistos negros) de la cual muy probablemente se contaminaron.

Palabras Clave: Adamelitas, peraluminoso, mesocrático-sódico.

\begin{abstract}
The post-orogenic magmatic intrusives localized in Tierra Caliente region, State of Mexico, at more or less $350 \mathrm{~km}$ of the Eocene-Miocene paleotrench, were emplaced in a continental crust with $35 \mathrm{~km}$ of thickness, characterized by black schist and Cretaceous volcanosedimentary rocks .

The individual typology of the stocks vary from adamelites to biotite granites. They belong to the mesocratic peraluminous domain, aluminous association with a high aluminosity index and, considering alkalinity, they belong to the sodic subtype. These geochemical characteristics are very similar to those determined for the Tizapa series (black schist) which very probably acted as contaminants of the samples under consideration.
\end{abstract}

Keywords: Adamelites, paraluminous, mesocratic-sodic.

\section{Introducción}

En la región de Tierra Caliente, Estado de México, los principales esfuerzos de cartografía general fueron realizados por Campa et al. (1974), Campa (1978), De Cserna y Fríes (1981) y De Cserna (1983). Algunos sectores con interés económico fueron analizados de manera más detallada por Colorado-Liévano (1979), DelgadoArgote et al. (1992), Tolson (1993) y Salinas-Priego (1994).

La relación entre los yacimientos minerales y la evolución tectónica ha sido discutida por Nieto-Obregón et al. (1977), González-Partida (1981, 1985) y Charoy y González-Partida (1984). Según González-Partida y Torres-Rodríguez (1988a), dos épocas magmáticas están directamente relacionadas con dos importantes tipos de mineralización en la región:

1) En el Cretácico una secuencia volcanosedimentaria contiene sulfuros masivos estratificados (GonzálezPartida 1981, 1984, 1985, 1993a, 1993b).

2) En el Eoceno-Mioceno el magmatismo se relaciona con mineralizaciones polimetálicas (VázquezMeneses y Vélez-Sánchez 1979; Salas-Castellanos 1982). 
Las edades radiométricas en Temascaltepec y Piedra Parada (de Cserna y Fries 1981) señalan una edad del Eoceno para estos dos cuerpos intrusivos.

El objetivo principal de este trabajo es presentar por primera vez datos geoquímicos de elementos mayores de los intrusivos de Piedra Parada, Amatepec (Tlatlaya), Temascaltepec y El Oro-Miahuatlán, cuya localización se muestra en la Figura 1.

\section{Características geoquímicas de los intrusivos}

Los granitos fueron durante mucho tiempo considerados como un grupo homogéneo independiente (Debon y Le Fort 1983; González-Partida y Torres-Rodríguez 1988b), donde las variaciones de las fases minerales (cuarzo-ortoclasa-plagioclasa, biotita, hornblenda, muscovita) en algunos casos daban problemas para una buena clasificación petrográfica. Las relaciones genéticas entre granitos y magmas de composición ácida a básica en una asociación plutónica ígnea han sido puestas en evidencia (Debon y Le Fort 1983), de manera análoga a las asociaciones volcánicas (series magmáticas) establecidas, así como la existencia de granitos derivados directamente de la anatexis. Los criterios de clasificación de los granitos pueden ser mineralógicos y/o químicos (para mayor detalle consultar a González-Partida y TorresRodríguez 1988b). Una clasificación químicomineralógica eficaz, bajo la forma de variables multicatiónicas que toma en cuenta todos los elementos mayores, son los diagramas químico-mineralógicos de Debon y Le Fort (1983), los cuales fueron usados para la clasificación de los granitos de Tierra Caliente. Las muestras colectadas en el campo, son representativas de los cuerpos intrusivos analizados.

\subsection{Clasificación químico-mineralógica}

A partir de los datos químicos de elementos mayores que se presentan en la Tabla 1, los óxidos fueron transformados a milicationes y luego a parámetros multielementales que revelan la naturaleza geoquímica de las rocas ígneas intrusivas de acuerdo a lo propuesto por Debon y Le Fort (1983). En los diagramas químicomineralógicos de estos autores, basados en los parámetros multielementales, primero se le da un nombre a la roca, luego se distingue la asociación magmática a la que pertenece (cafémica, alumino-cafémica y aluminosa), en donde algunos subtipos pueden aparecer.

El análisis catiónico de los parámetros $Q-P$ (donde: $Q=\mathrm{Si} / 3-(\mathrm{K}+\mathrm{Na}+2 \mathrm{Ca} / 3)$ y $P=\mathrm{K}-(\mathrm{Na}+\mathrm{Ca}))$ permite darle un nombre a la roca y están expresados en gramosátomo x $10^{3}$ por cada elemento en $100 \mathrm{~g}$ de muestra.

En la Figura 2 se muestran graficados los resultados de los análisis geoquímicos de los cuatro troncos granitoides. En Miahuatlán se presentan las fases de cuarzomonzodiorita, granodiorita, adamelita y granito. En
Amatepec (Tlatlaya) están presentes las fases granodioríticas, principalmente adamelita y granito. En Temascaltepec y Piedra Parada los troncos son composicionalmente más homogéneos, correspondiendo a adamelitas.

\subsection{Asociación magmática de los intrusivos}

La Figura 3 está construida a partir de los parámetros $A-B$ (donde $A=\mathrm{Al}-(\mathrm{K}+\mathrm{Na}+2 \mathrm{Ca})$ y $B=\mathrm{Fe}+\mathrm{Mg}+\mathrm{Ti}$ ) y manifiesta el grado de aluminosidad de una asociación (o serie) magmática, los sectores I, II y III corresponden al dominio peraluminoso, y los sectores IV, V y VI al metaluminoso. Las rocas ígneas que presentan una alta aluminosidad pueden estar contaminadas debido a su emplazamiento cortical, y su medio ambiente directo puede ser la respuesta a dicha contaminación. En la misma Figura 3 se han graficado los resultados de los análisis químicos de roca total de los esquistos de Tizapa publicados por González-Partida (1993b). En base a las tendencias, se sugiere que los troncos del Eoceno se pudieron contaminar con esquistos aluminosos.

Los intrusivos de Tierra Caliente se encuentran dentro de los granitoides paraluminosos, este índice de aluminosidad es estimado a partir del parámetro $A$ y se dice que una asociación es muy baja en aluminio cuando este parámetro es menor que 10, o muy alta cuando es mayor que 60. Las asociaciones peraluminosas generalmente son ricas en micas y se pueden distinguir varios sub-tipos, de los cuales el primero considera el contenido de cuarzo. Así en la Figura 4, el diagrama ternario $Q-B-F$ presenta una línea crítica, por arriba de la cual se encuentran los granitoides con alto contenido de cuarzo, los muy próximos a ella son normales en cuarzo y los que están por debajo son pobres en cuarzo. Como se puede apreciar, los intrusivos estudiados son ligeramente ricos en cuarzo, presentando una tendencia general a la evolución de las fases mineralógicas presentes. El índice de color (leucocrático, subleucocrático, mesocrático), igualmente marcado en la Figura 4, indica que los intrusivos son de la asociación mesocrática. Los granitoides peraluminosos mesocráticos presentan un rango de alcalinidad que varía de potásico, sódico-potásico a sódico, de acuerdo a los parámetros que se muestran en la Figura 5. Los intrusivos considerados en este estudio pertenecen a la asociación mesocrática-sódica. A manera de resumen se puede decir que los intrusivos del Eoceno del Estado de México son adamelitas peraluminosas mesocrático-sódicas.

En la Figura 6 se presenta la relación $\mathrm{SiO}_{2}$ vs. $\mathrm{K}_{2} \mathrm{O}$. Se han graficado los resultados de los troncos, así como el comportamiento de los esquistos negros de Tizapa y de la serie volcanosedimentaria cretácica que atraviesan.

De acuerdo a lo expuesto en la Figura 6, los troncos corresponderían a la serie calcialcalina, a diferencia de las rocas volcanosedimentarias que presentan una tendencia bimodal de alcalina a calcialcalina en los miembros más diferenciados. Los esquistos aluminosos 


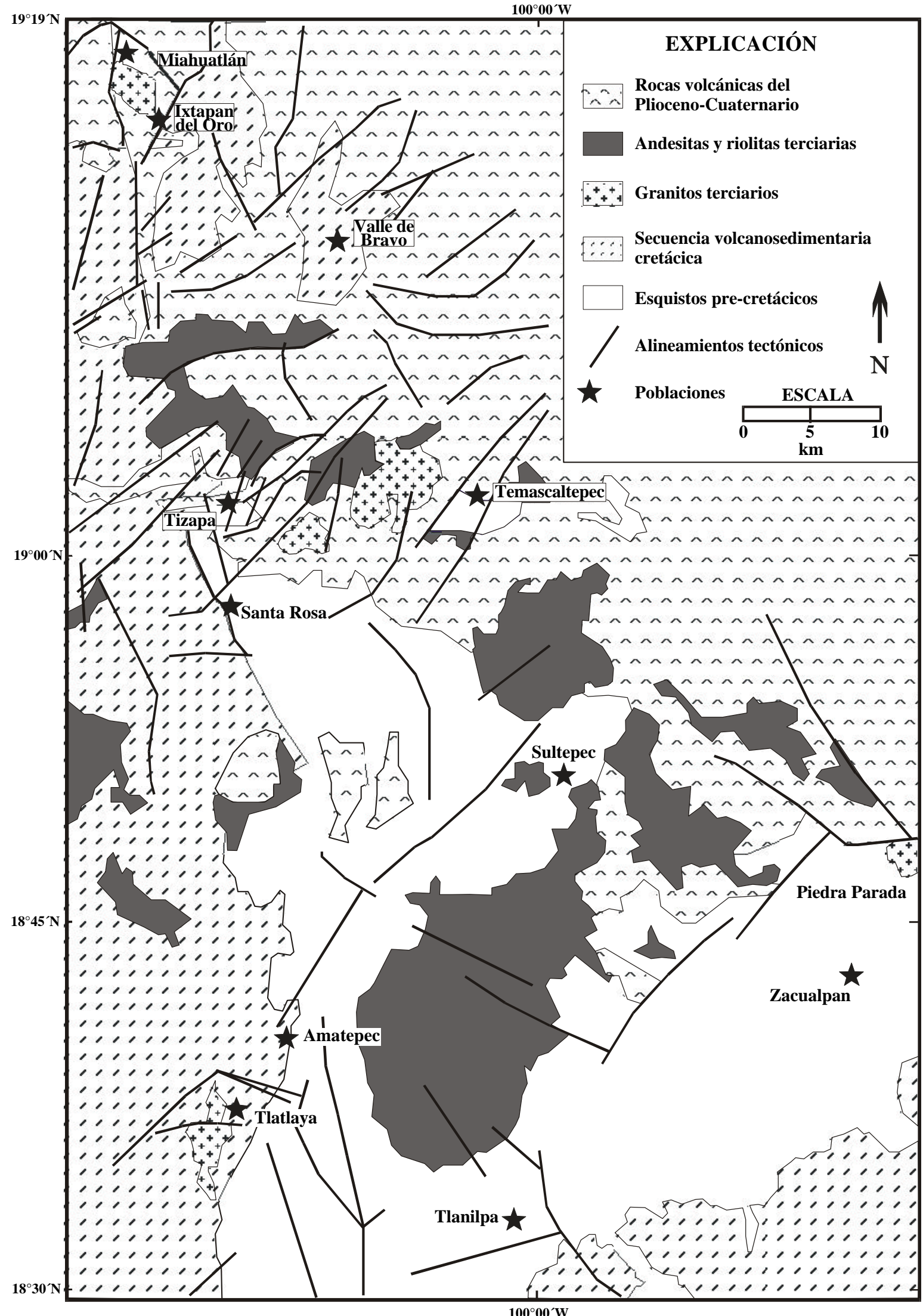

Figura 1. Esquema geológico general de la región de Tierra Caliente, Estado de México, que muestra la localización de los cuerpos intrusivos. Tomado de González-Partida (1981).. 
de Tizapa, calcialcalinos en este diagrama, se enmarcan dentro de la misma tendencia que los troncos del Eoceno.

\section{Potencialidad metálica de los granitoides: discusión}

De acuerdo a la tectónica global es posible asociar los granitos a diversos ambientes geodinámicos, en cada uno de los cuales se presenta una composición que los distingue: en los arcos insulares se presentan los granitos toleíticos y calcialcalinos; en márgenes continentales activas, granitos calcialcalinos y calcialcalinos potásicos; en zonas de colisión continental granitos aluminosos; en cratones estables granitos peraluminosos; y en dominios oceánicos, plagiogranitos (González-Partida et al. 1987). La gran variedad de tipos de granitos asociados a ambientes tectónicos particulares ha generado una diversidad de nomenclaturas surgidas de diferentes criterios químicos, mineralógicos, petrográficos, isotópicos y de especialidad metálica (Atherton y Tarney 1981; Debon y Le Fort 1983).

Los grupos de granitos cafémicos (y/o tipo I, tipo magnetita, calcialcalinos y subalcalinos), conteniendo magnetita + biotita como accesorios, se forman por fusión parcial de la corteza profunda y/o manto superior, o por anatexis de metasedimentos poco maduros; son magmas contaminados o híbridos, presentan una relación de $\mathrm{Sr}$ baja, y se relacionan a vetas y skarns de $\mathrm{Mo}, \mathrm{Cu}, \mathrm{y}$ W (Meinert 1995). Estos magmas presentan una alta fugacidad de oxígeno y una relación alta en su tendencia

Tabla 1. Análisis químicos de roca total de los intrusivos de Miahuatlán (MIA), Temascaltepec (TEM), Piedra Parada (PIE) y Amatepec (AMA). Los parámetros: Q, P, A, B, F, G, H e I fueron propuestos por Debon y Le Fort (1983), y para su cálculo se emplearon los datos de óxidos mayores transformados a milicationes. P.I. = perdida por ignición. Los análisis fueron hechos en el CRPG-CNRS de Nancy, Francia.

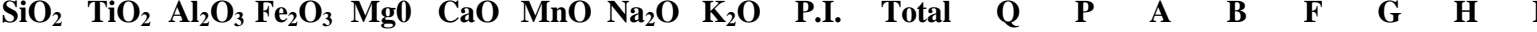

\begin{tabular}{|c|c|c|c|c|c|c|c|c|c|c|c|c|c|c|c|c|c|c|c|}
\hline \multicolumn{20}{|c|}{ MIAHUATLÁN } \\
\hline MIA.1 & 63.3 & 0.23 & 19.2 & 4.6 & 2.48 & 2.29 & 0.00 & 3.1 & 3.33 & 1.40 & 99.60 & 154 & -70 & 124 & 123 & 279 & 171 & 0.41 & 0.52 \\
\hline MIA.2 & 54.6 & 0.52 & 21.0 & 7.9 & 3.69 & 5.89 & 0.00 & 3.2 & 1.78 & 1.86 & 100.00 & 92 & -171 & 60 & 197 & 266 & 141 & 0.27 & 0.48 \\
\hline MIA.3 & 54.8 & 0.49 & 21.5 & 7.7 & 3.48 & 5.43 & 0.04 & 3.5 & 1.76 & 1.40 & 100.10 & 90 & -172 & 78 & 189 & 276 & 150 & 0.25 & 0.48 \\
\hline MIA.4 & 66.0 & 0.19 & 21.5 & 2.4 & 1.35 & 0.66 & 0.06 & 2.6 & 3.39 & 1.70 & 99.85 & 203 & -24 & 242 & 66 & 286 & 156 & 0.46 & 0.53 \\
\hline TEM.1 & 66.1 & 0.24 & 20.4 & 3.0 & 1.34 & 1.55 & 0.09 & 3.0 & 3.10 & 1.30 & 100.12 & 186 & -58 & 182 & 74 & 295 & 163 & 0.41 & 0.47 \\
\hline TEM.2 & 66.7 & 0.19 & 20.9 & 2.8 & 1.26 & 1.50 & 0.08 & 2.9 & 3.10 & 0.80 & 100.23 & 193 & -54 & 197 & 69 & 293 & 160 & 0.41 & 0.47 \\
\hline TEM.3 & 68.4 & 0.03 & 21.5 & 1.2 & 0.68 & 0.42 & 0.07 & 2.5 & 3.63 & 1.50 & 99.55 & 217 & -11 & 249 & 33 & 305 & 158 & 0.49 & 0.53 \\
\hline TEM.4 & 65.2 & 0.26 & 20.5 & 3.3 & 1.38 & 1.59 & 0.02 & 3.5 & 3.20 & 1.10 & 100.05 & 163 & -73 & 164 & 79 & 313 & 181 & 0.38 & 0.45 \\
\hline TEM.5 & 67.0 & 0.25 & 19.0 & 3.6 & 1.42 & 1.90 & 0.03 & 2.8 & 3.32 & 0.80 & 100.12 & 189 & -54 & 143 & 84 & 282 & 161 & 0.44 & 0.44 \\
\hline TEM.7 & 65.5 & 0.25 & 20.0 & 3.4 & 1.40 & 1.80 & 0.04 & 3.2 & 3.54 & 0.80 & 99.93 & 164 & -60 & 142 & 81 & 310 & 179 & 0.42 & 0.45 \\
\hline TEM.8 & 66.6 & 0.25 & 18.1 & 3.5 & 1.27 & 2.00 & 0.05 & 3.3 & 3.29 & 1.60 & 99.96 & 170 & -72 & 106 & 78 & 307 & 176 & 0.40 & 0.42 \\
\hline TEM.9 & 66.2 & 0.21 & 19.8 & 3.1 & 1.60 & 1.67 & 0.03 & 2.1 & 3.88 & 1.60 & 100.19 & 198 & -15 & 177 & 81 & 276 & 150 & 0.55 & 0.51 \\
\hline TEM.10 & 67.0 & 0.22 & 18.3 & 3.4 & 1.36 & 1.89 & 0.05 & 3.4 & 3.58 & 0.70 & 99.90 & 164 & -67 & 105 & 79 & 312 & 186 & 0.41 & 0.44 \\
\hline \multicolumn{20}{|c|}{ AMATEPEC } \\
\hline AMA.1 & 65.1 & 0.26 & 18.2 & 4.8 & 1.42 & 2.09 & 0.04 & 3.0 & 3.69 & 1.65 & 100.25 & 161 & -56 & 108 & 94 & 295 & 175 & 0.45 & 0.37 \\
\hline AMA. 2 & 65.6 & 0.30 & 17.8 & 5.4 & 1.56 & 2.01 & 0.06 & 2.6 & 3.71 & 1.80 & 99.25 & 178 & -41 & 114 & 110 & 267 & 163 & 0.48 & 0.37 \\
\hline AMA.3 & 59.7 & 0.37 & 18.6 & 7.0 & 2.55 & 4.06 & 0.08 & 3.5 & 2.73 & 1.40 & 99.99 & 113 & -127 & 49 & 155 & 287 & 171 & 0.34 & 0.42 \\
\hline AMA.4 & 64.2 & 0.23 & 19.6 & 4.3 & 1.27 & 2.84 & 0.08 & 3.2 & 3.74 & 0.50 & 99.96 & 152 & -57 & 97 & 88 & 315 & 183 & 0.44 & 0.37 \\
\hline \multicolumn{20}{|c|}{ PIEDRA PARADA } \\
\hline PIE.3 & 65.8 & 0.19 & 18.5 & 3.2 & 1.44 & 1.90 & 0.05 & 3.5 & 3.33 & 1.40 & 99.31 & 159 & -76 & 111 & 79 & 317 & 184 & 0.39 & 0.97 \\
\hline PIE.4 & 69.0 & 0.14 & 17.1 & 2.6 & 1.06 & 1.50 & 0.07 & 3.9 & 3.55 & 1.10 & 100.02 & 164 & -77 & 81 & 61 & 330 & 201 & 0.38 & 0.45 \\
\hline
\end{tabular}

$\mathrm{Q}=\mathrm{Si} / 3-(\mathrm{K}+\mathrm{Na}+2 \mathrm{Ca} / 3) ; \quad \mathrm{P}=\mathrm{K}-(\mathrm{Na}+\mathrm{Ca}) ; \quad \mathrm{A}=\mathrm{Al}-(\mathrm{K}+\mathrm{Na}+2 \mathrm{Ca}) ; \mathrm{B}=\mathrm{Fe}+\mathrm{Mg}+\mathrm{Ti} ; \quad \mathrm{F}=555-(\mathrm{Q}+\mathrm{B}) ; \quad \mathrm{G}=\mathrm{K}+\mathrm{Na} ;$

$\mathrm{H}=\mathrm{K} /(\mathrm{Na}+\mathrm{K}) ; \quad \mathrm{I}=\mathrm{Mg} /(\mathrm{Fe}+\mathrm{Mg})$. 


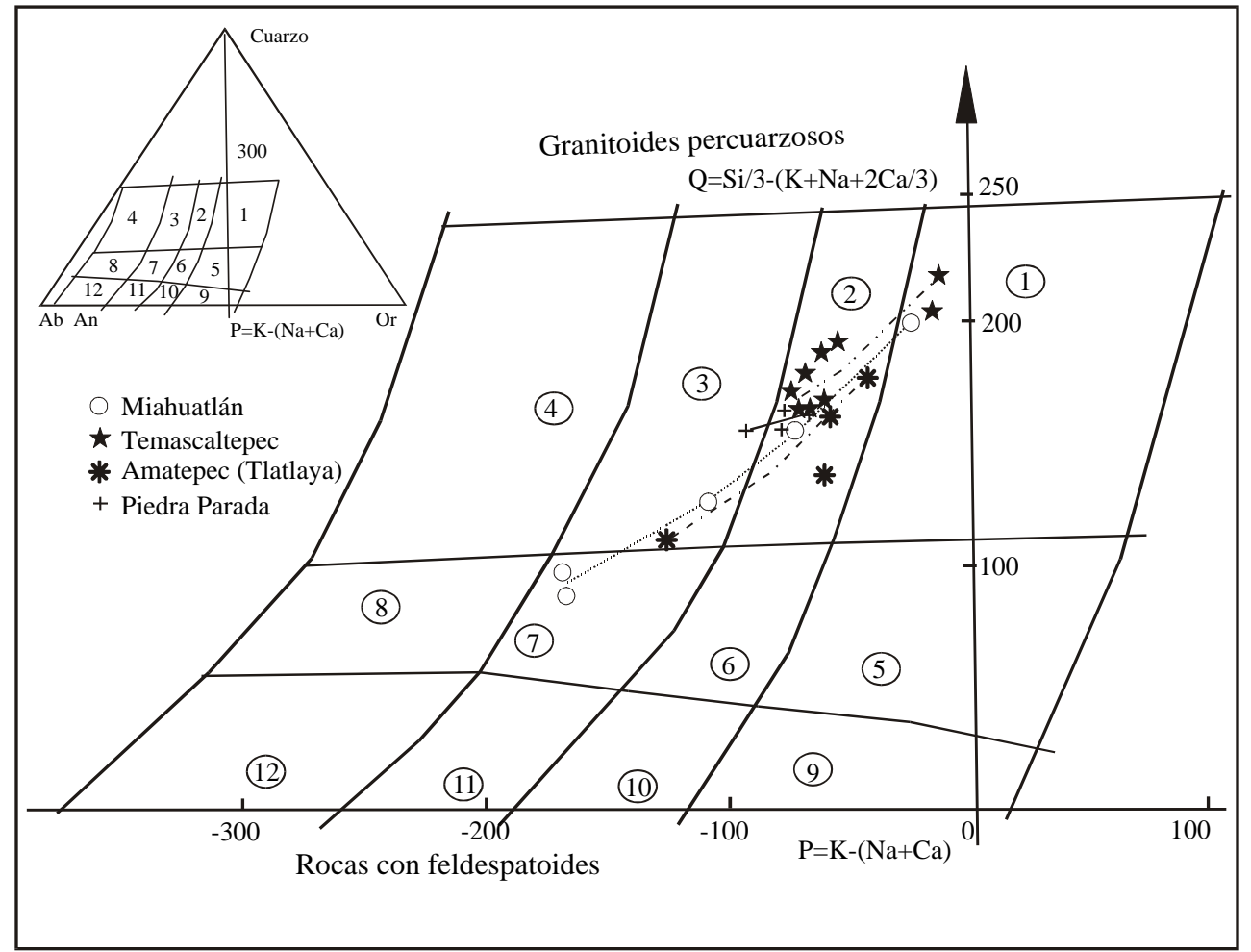

Figura 2. Diagrama Q - P de Debon y Le Fort (1983) que permite clasificar las rocas ígneas de acuerdo a su composición química de elementos mayores, transformados a milicationes, donde: 1: granito, 2: adamelita, 3: granodiorita, 4: tonalita, 5: cuarzo-sienita, 6: cuarzomonzonita, 7: cuarzomonzodiorita, 8: cuarzodiorita, 9: sienita, 10: monzonita, 11: monzogabro, 12: gabro.

férrico/ferrosa, se forman en ambientes tectónicos orogénicos a postorogénicos, arcos insulares y márgenes continentales; las relaciones isotópicas presentan valores de $\delta^{34}$ S entre -3.7 y $+4.2 \%$ y de $\delta^{18} \mathrm{O}$ entre +6 y $+10 \%$ o (Atherton y Tarney 1981).

Por otro lado, los granitos aluminopotásicos (y/o tipo $\mathrm{S}$, tipo ilmenita) contienen biotita, muscovita e ilmenita como accesorios, su fuente es la corteza superior o inferior, se pueden presentar condiciones de anatexis diferencial a partir de metasedimentos, presentándose en áreas con un gradiente geotérmico regional elevado, pueden igualmente presentarse como mezcla de plutones removilizados, están saturados en agua, presentan relaciones isotópicas de estroncio inicial superiores a los tipos anteriores (cafémicos), y presentan una mayor fertilidad metálica: Sn, W, U, Ba, Au, F, Nb-Ta-Cs, (González-Partida et al. 1987; Meinert 1995). Los magmas presentan una baja fugacidad de oxígeno y una relación baja en la relación férrico/ferrosa caracterizado por la presencia de ilmenita; las relaciones isotópicas de azufre varían de $\delta^{34} \mathrm{~S}=-5.7$ hasta $-10 \%$ y las de oxígeno son de $\delta^{18} \mathrm{O}=+10 \%$ (Atherton y Tarney 1981). Su ambiente tectónico es sinorogénico a orogénico tardío, dominios corticales anatécticos y de colisión continental.

En Tierra Caliente la corteza continental tiene un espesor de $\sim 35 \mathrm{~km}$ (Urrutia-Fucugauchi 1986; MolinaGarza y Urrutia-Fucugauchi 1993) y se encuentra a 350 $\mathrm{km}$ de la paleotrinchera del Eoceno-Mioceno y el magmatismo intrusivo cortó esquistos y rocas volcanosedimentarias de donde muy probablemente se contaminaron adquiriendo su carácter peraluminoso mesocrático (y/o tipo S, tipo ilmenita). Datos isotópicos de azufre obtenidos por González-Partida (1981), concuerdan con la interpretación.

Las rocas intrusivas eocénicas del Estado de México están emplazadas en una corteza continental de $35 \mathrm{~km}$ de espesor (Urrutia-Fucugauchi 1986; Molina-Garza y Urrutia-Fucugauchi 1993), y hacia la costa, en donde se encuentran los yacimientos de cobre-oro, disminuye a 30 $\mathrm{km}$, y a $20 \mathrm{~km}$ en el cinturón ferrífero de la costa occidental mexicana. Según Kesler (1997), el incremento de sílice en los magmas está directamente relacionado con el espesor de la corteza en márgenes convergentes, y muchos yacimientos igualmente tienen un control similar. Así, el estaño se encuentra en cortezas continentales con espesores superiores a los $50 \mathrm{~km}$, la fluorita, mercurio y antimonio comparten las mismas características. Pero en Tierra Caliente los patrones no muestran estas tendencias, ya que la corteza no sobrepasa los $35 \mathrm{~km}$ de espesor. De hecho, Clark et al. (1982) determinaron zonas subparalelas a la trinchera en donde el magma y la mineralización están espacial y temporalmente relacionados; los yacimientos de $\mathrm{Cu}-$ (Mo-W) se relacionan con magmas calcialcalinos con 

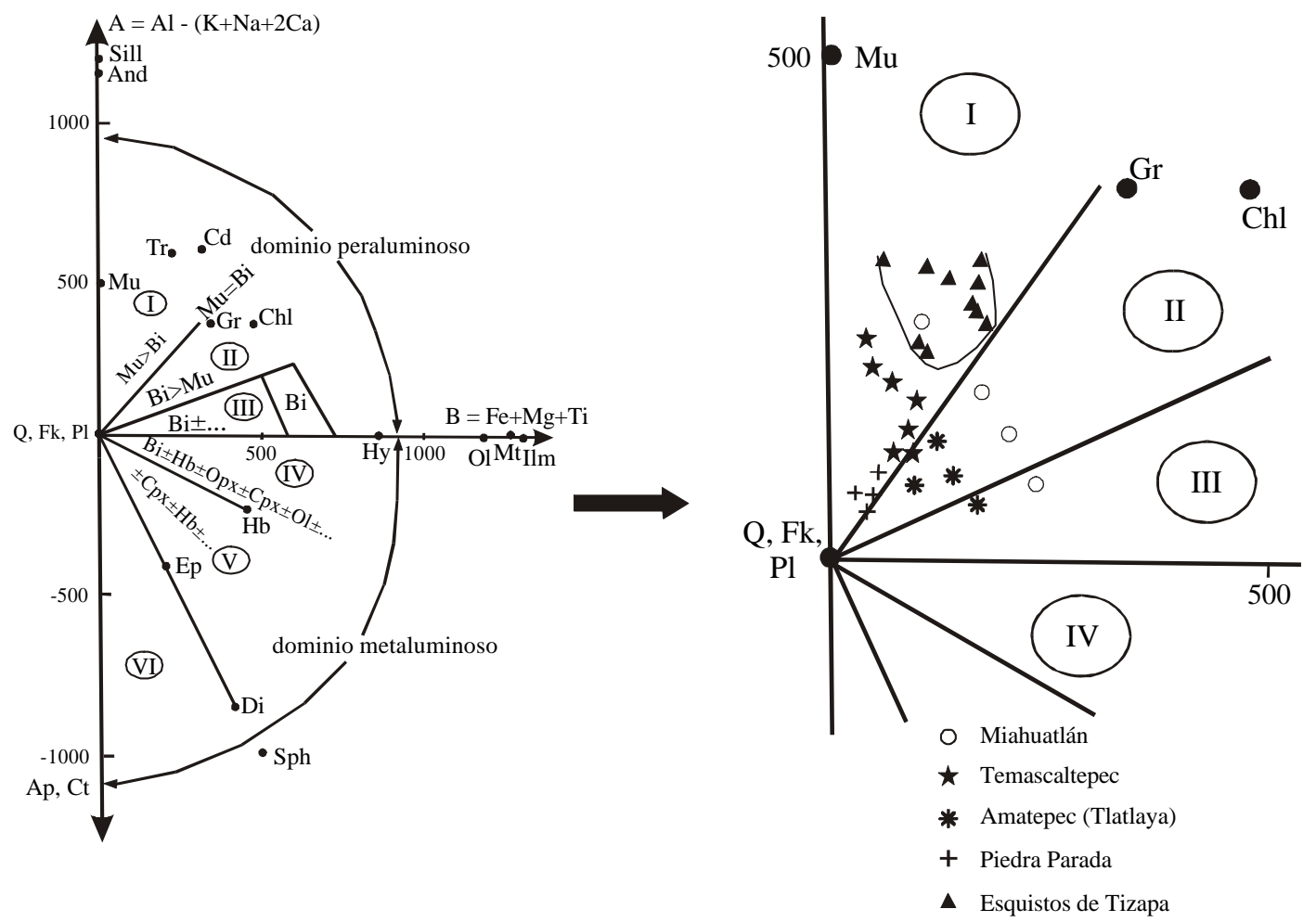

Figura 3. Diagrama A - B de Debon y Le Fort (1983), que permite determinar el grado de aluminosidad de una roca ígnea. Los datos de las metapelitas del esquisto de Tizapa, fueron tomados de González-Partida (1993a).

edades que van de los 106 a $40 \mathrm{Ma}$, el $\mathrm{CaF}_{2}$ (fluorita) coincide con la provincia magmática alcalina y el $\mathrm{Pb}-\mathrm{Zn}$ Ag se encuentra en las partes más internas de la zona de subducción entre los 49 y 26 Ma. Según Clark et al. (1982), las variaciones geodinámicas, más las características isotópicas de $\mathrm{Sr}, \mathrm{Rb}$ y $\mathrm{S}$ sugieren que, junto con la subducción, procesos de contaminación cortical debieron haber participado en la distribución de los cinturones metálicos.

Estudios de metalogenia regional relacionados con la convergencia de placas, indican que la distribución metálica es un reflejo del arco magmático y su distribución espacial en el tiempo (Kesler 1978; Mitchell y Garson 1981; Sillitoe 1989). Entre la costa occidental y Tierra Caliente, la migración lateral del magmatismo continental ocurrido entre el Cretácico Tardío y el Terciario medio definieron las provincias metálicas del centro-occidente de México (González-Partida 1981, 1985; González-Partida y Torres-Rodríguez 1988a). Las asociaciones más importantes son: Fe(Ti) en skarns: Las Truchas, Michoacán; $\mathrm{Cu}-\mathrm{Au}$ (brechas, pórfidos, diseminados): La Verde e Inguarán, Michoacán; $\mathrm{Pb}-\mathrm{Zn}-\mathrm{Ag}$ (Au-Cu): Taxco-Zacualpán-Temascaltepec; $\mathrm{CaF}_{2}$ : La Azul, Gro., Zacualpan Estado de Mex.; Mn, Hg-Sb en vetas: Huitzuco, Gro. (González-Partida y TorresRodríguez 1988a). En el Estado de México, los troncos intrusivos son en parte la roca encajonante de las vetas polimetálicas, como se observa en Temascaltepec y Amatepac-Tlatlaya. En Taxco, el magmatismo relacionado a la mineralización es del Oligoceno (MoránZenteno et al. 1998). El cinturón polimetálico vetiforme que se extiende a lo largo de Taxco, Zacualpan, Sultepec, Temascaltepac, El Oro-Miahuatlán, Angangueo y El Oro-Tlalpujahua se relaciona al Oligoceno, época de gran fertilidad metálica en México, (VázquezMeneses y Vélez-Sánchez 1979; Salas-Castellanos 1982; González-Partida y Torres-Rodríguez 1988a).

\section{Conclusiones}

La tipología individual de los troncos del Eoceno del Estado de México, los define como adamelitas y granitos de biotita que pertenecen al dominio peraluminoso mesocrático, asociación aluminosa, con un índice de aluminosidad alto y son normales en cuarzo. Por su rango de alcalinidad pertenecen al subtipo sódico, estas características geoquímicas son muy similares a las determinadas en la serie Tizapa de la cual muy probablemente se contaminaron.

Los intrusivos en el Estado de México son en parte la roca encajonante de las vetas polimetálicas, como se observa en Temascaltepec y Amatepec-Tlatlaya, y presentan fertilidad metálica acreditada espacialmente, 


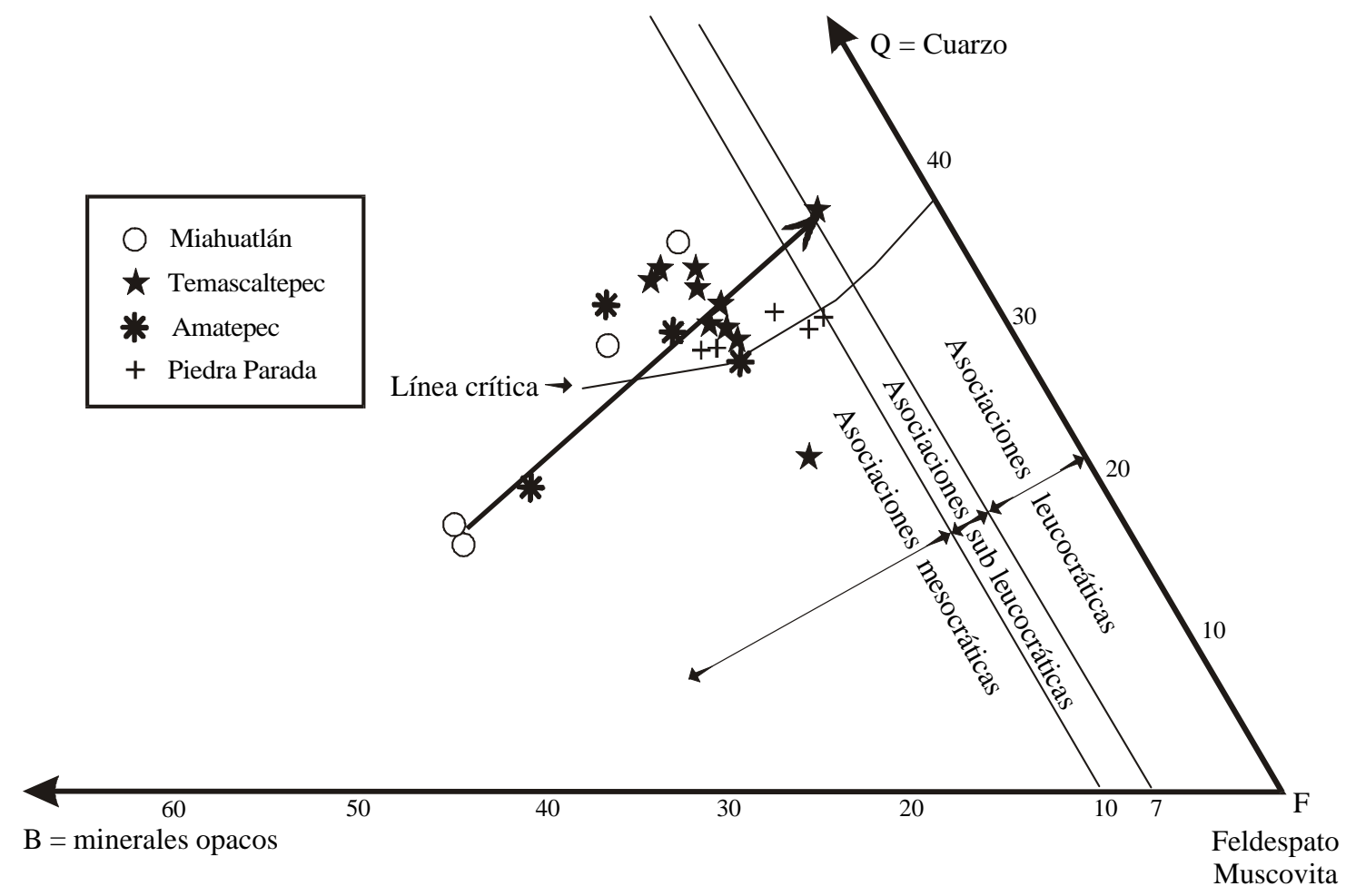

Figura 4. Clasificación Q-B-F de las asociaciones aluminosas de Debon y Le Fort (1983).

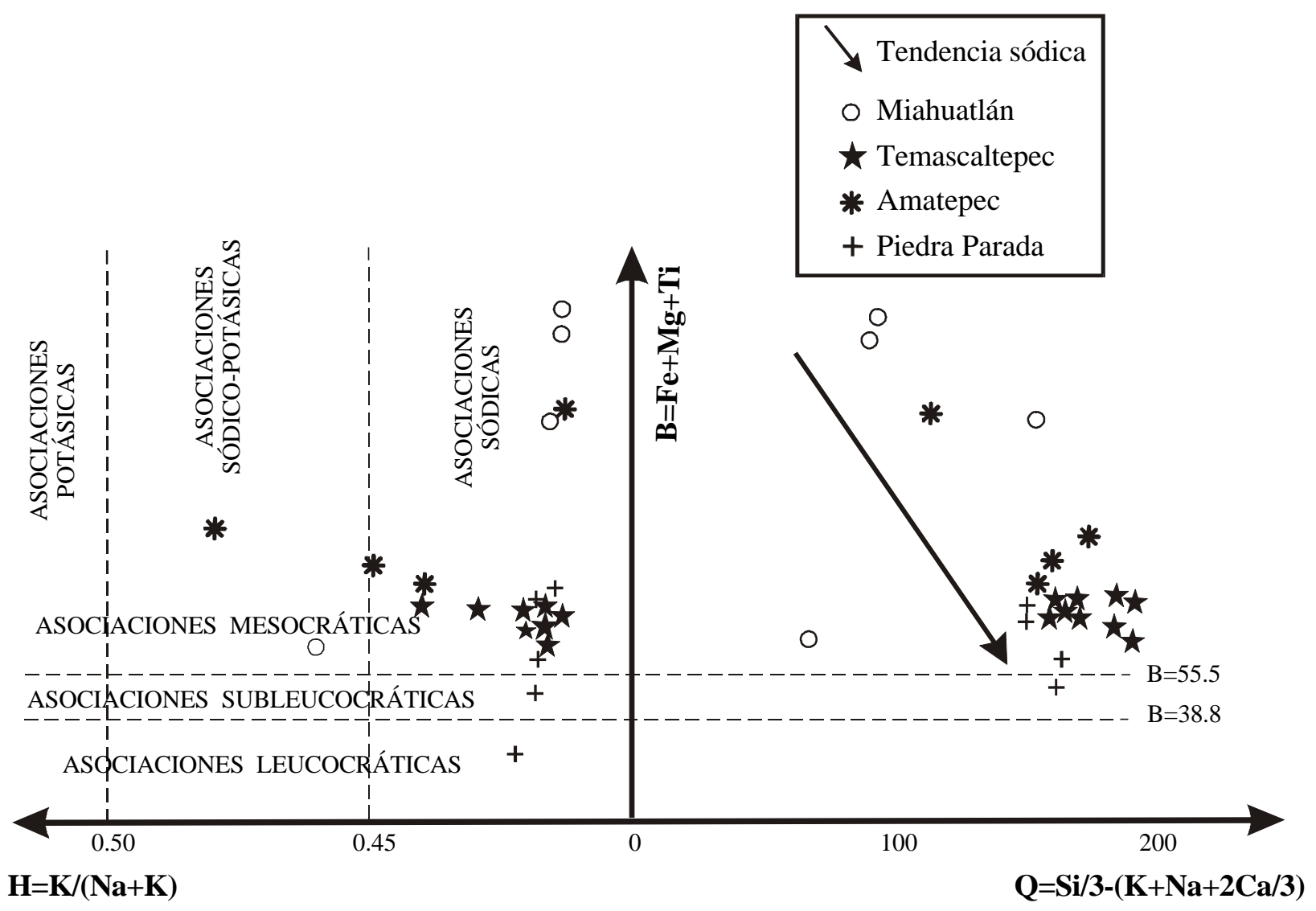

Figura 5. Clasificación de la asociación aluminosa usando los parámetros B-H,Q de Debon y Le Fort (1983). 


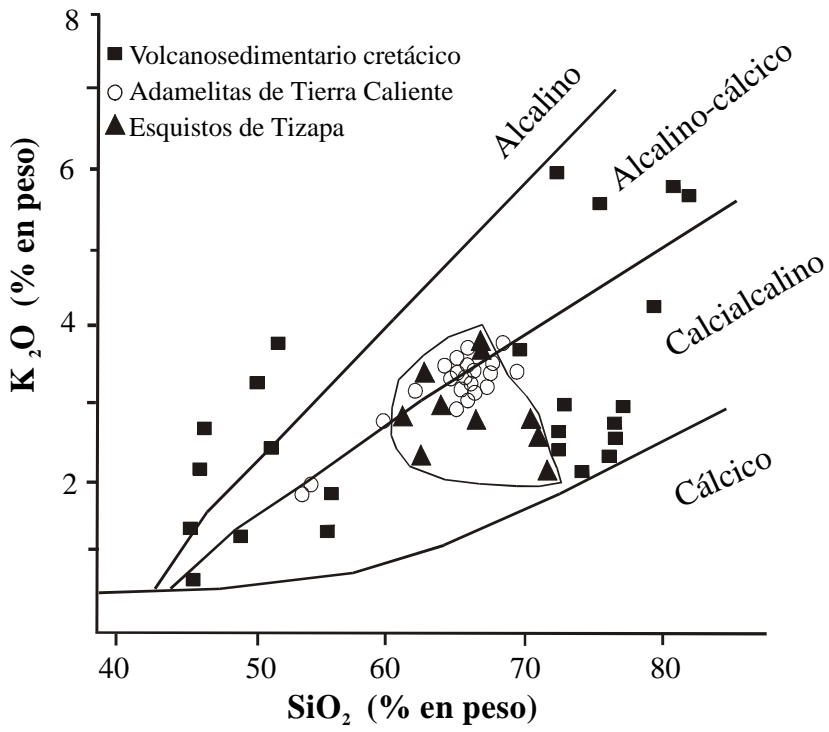

Figura 6. Diagrama clásico $\mathrm{K}_{2} \mathrm{O}-\mathrm{SiO}_{2}$ que muestra los campos de las series magmáticas alcalina, alcalino-cálcica, calcialcalina y cálcica, tomado de González-Partida y Torres-Rodríguez (1988b). Los datos de los esquistos negros de Tizapa y rocas volcanosedimentarias cretácicas fueron tomados de González-Partida (1993a, 1993b).

así como una distribución a lo largo del cinturón polimetálico vetiforme del Oligoceno que se extiende regionalmente de Taxco, Estado de Guerrero hacia el norte a El Oro-Tlalpujahua, Estado de México.

\section{Agradecimientos}

Agradecemos el apoyo de los editores, en particular a la Dra. Susana Alaniz Álvarez y a la Dra. Ma. Teresa Orozco Esquivel. El M. en C. Mariano Elías Herrera contribuyó con sus atinadas observaciones. La M. en C. Mireya Maples, la Lic. T. Soledad Medina Malagón, el Sr. Crescencio Garduño y Juan T. Vázquez dieron siempre gentilmente su apoyo. Este trabajo forma parte de las investigaciones referentes a la Relación entre Magma y Depósitos Minerales que se realizan en la Unidad de Ciencias de la Tierra del Instituto de Geología de la UNAM, las cuales son apoyadas por el CONACYT Proyecto No. 32511-T.

\section{Referencias Bibliográficas}

Atherton, M.P., Tarney, J., 1981, Origin of granite batholiths. Geochemical evidence: Shiva Publishing Ltd., Cheshire, England, $148 \mathrm{p}$.

Campa, M.F., 1978, La evolución tectónica de Tierra Caliente, Gro.: Boletín de la Sociedad Geológica Mexicana, 39, 52-64.

Campa, M.F., Campos, R., Flores, R., Oviedo, R., 1974, La secuencia mesozoica volcanosedimentaria metamorfizada de Ixtapan de la Sal México-Teloloapan Guerrero: Boletín de la Sociedad Geológica Mexicana, 35, 7-28.

Charoy, B., González-Partida, E., 1984, Analysis des phases fluides associees a la genese des amas sulfures et des filons Au-Ag de la
Province Taxco-Guanajuato Mexique: Bulletin de Mineralogie, 107, 285-305.

Clark, K.F., Foster, C.T., Damon, P.E., 1982, Cenozoic mineral deposits and subduction-related magmatic arcs in México: Geological Society of America Bulletin, 93, 533-544.

Colorado-Liévano, D., 1979, Etude de une partie de la ceinture metamorphique (Mesozoique) du Sud de Mexique (Etats de México et Guerrero): These 3e Cicle, Universite Pierre et Marie Curie, Paris, France, $130 \mathrm{p}$.

Debon, F.P., Le Fort, P., 1983, A chemical mineralogical classification of common plutonic rocks and associations: Transactions of the Royal Society of Edinburgh - Earth Science, 73, 135-149.

De Cserna, Z., 1983, Hoja Tejupilco 14 Q-g (9) con resumen de la geología de la hoja Tejupilco: Universidad Autónoma de México, Instituto de Geología, Cartas Geológicas de México serie de 1: 100,000, núm. 18, 1 mapa con texto, 28 p.

De Cserna, Z. Fries, C., 1981, Hoja Taxco 14Q-h (7) con resumen de la geología de la hoja Taxco, Edos de Gro., México, y Mich.: Universidad Autónoma de México, Instituto de Geología, Cartas Geológicas de México serie 1: 100,000, 1 mapa con texto, 47 p.

Delgado-Argote, L., López-Martinez, M., York, D., Hall, C.M., 1992, Geologic framework and geochronology of ultramafic complexes of southern México: Canadian Journal of Earth Sciences, $29,1590-1604$.

González-Partida, E., 1981, La province filoniene Au-Ag de Taxco Guanajuato (Mexique): Caracteristiques des solutions hydrothermales, composition isotopique du soufre et proposition de un modele matallogenique sur le exemple del filons de Miahuatlan, Sultepec et Temascaltepec: Centro de Investigaciones Petrográficas y Geoquímicas y Escuela Nacional de Geología CRPG-ENSG, Nancy, Francia, These DocteurIngenieur, $235 \mathrm{p}$.

González-Partida, E., 1984, Análisis de las inclusiones fluidas e isótopos de azufre, hidrógeno y oxígeno de los sulfuros masivos de Tizapa- Sta Rosa, Edo. de Méx: GEOMIMET, 128, 66-76.

González-Partida, E., 1985, Etude metallogenique de la pertie CentroOccidentale du Mexique: Instituto Nacional Politécnico de Loren y Centro Nacional de Investigación Científica INPL-CNRS, Nancy, Francia, These de Doctorat de Etat, 330p.

González-Partida, E., 1993a, Petrografía, geoquímica, cristaloquímica y características de los fluidos asociados a una sección del metamórfico aflorante en Tierra Caliente, Estados de México, Guerrero y Michoacán: Geofísica Internacional, 32, 429-440.

González-Partida, E., 1993b, Datos geoquímicos de roca total para la secuencia vulcanosedimentaria cretácica en Cuale-El Rubí, Jalisco y Tierra Caliente, Guerrero, México: Geofísica Internacional, $32,249-259$

González-Partida, E., Torres-Rodríguez, V., 1988a, Evolución tectónica de la porción centro-occidental de México y su relación con los yacimientos minerales: Geofísica Internacional, 27(4), 543581.

González-Partida, E., Torres-Rodríguez, V., 1988b, Petrogénesis de rocas ígneas: Universidad Nacional Autónoma de México, Facultad de Ingeniería, Apuntes de la División de Estudios de Posgrado (DEPFI), $216 \mathrm{p}$.

González-Partida, E., Torres-Rodríguez, V., Stussi, J.M., Zimmermann, J.L., 1987, Análisis geoquímico de los granitoides aflorantes en el sector Puerto Vallarta-Río de Santiago, México: Litósfera, 1, 33-49.

Kesler, S.E, 1978, Metallogenesis of the Caribbean region: Journal of the Geological Society, 135, 429-441.

Kesler, S.E., 1997, Metallogenic evolution of convergent margins: Selected ore deposit models: Ore Geology Reviews, 12, 153-171.

Meinert, L.D., 1995, Compositional variation of igneous rocks associated with skarn deposits - Chemical evidence for a genetic connection between petrogenesis and mineralization, in Thompson J.F.H. (ed.), Magmas, fluids, and ore deposits: Mineraralogical Association of Canada, Short course, 23, 401-418.

Molina-Garza, R., Urrutia-Fucugauchi, J., 1993, Deep crustal structure 
of central Mexico derived from interpretation of Bouguer gravity anomaly data: Journal of Geodynamics, 17(4), p.181-201.

Morán-Zenteno, D.J., Alba-Aldave, L.A., Martínez-Serrano, R.G., Reyes-Salas, M.A., Corona-Esquivel, R., Ángeles-García, S., 1998, Stratigraphy, geochemistry and tectonic significance of the Tertiary volcanic sequences of the Taxco-Quetzalapa region, southern Mexico: Revista Mexicana de Ciencias Geológicas, 15(2), 167-180.

Mitchell, A.H.G. Garson, M.S., 1981, Mineral deposits and global tectonic settings: Academic Press, New York, $326 \mathrm{p}$

Nieto-Obregón, J., Cruz-Rios, R., Colorado-Liévano, D., FigueroaPalacios, M., Franco-Serrato, E., González-Partida, E., 1977, Elementos tectónicos y metalógeneticos para considerar el potencial económico-minero de la región comprendida entre Zacualpan y El Oro, Edo. de México: Consejo de Recursos Minerales, Boletín, 6, 644-667.

Salas-Castellanos, J.E., 1982, Geología de la región de Amatepec y evaluación matalogenética del prospecto "La Sierrita", Tlatlaya,
Edo. de México: Universidad Nacional Autónoma de México, Facultad de Ingeniería, Tesis profesional, $101 \mathrm{p}$.

Salinas-Priego, J.C., 1994, Etude structurale du sud-ouest Mexicain (Guerrero): Analyse microtectonique des deformations ductiles du Tertiaire inferieur: Tesis doctoral, Universite de Orleans, Francia, Memoria No. 16, 228 p.

Sillitoe, R.H., 1989, Gold deposits in the western Pacific - The magmatic connection: Economic Geology, 6, 274-291.

Tolson, G., 1993, Structural geology and tectonic evolution of the Santa Rosa area, SW Mexico State, Mexico: Geofísica Internacional, 32, 397-414

Urrutia-Fucugauchi, J., 1986, Crustal thickness, heat flow, arc magmatism and tectonics of Mexico -preliminary report: Geofísica Internacional, 25(4), 559-573.

Vázquez-Meneses, E., Vélez-Sánchez, V.C., 1979, Estudio geológico minero del área Valle de Bravo, México: Universidad Nacional Autónoma de México, Facultad de Ingeniería, Tesis profesional, $86 \mathrm{p}$. 